\title{
O-star mass-loss rates at low metallicity
}

\author{
L. B. Lucy
}

\begin{abstract}
Astrophysics Group, Blackett Laboratory, Imperial College London, Prince Consort Road, London SW7 2AZ, UK e-mail: 1.1ucy@imperial.ac.uk
\end{abstract}

Received 18 April 2012 / Accepted 11 May 2012

\section{ABSTRACT}

\begin{abstract}
Mass fluxes $J$ are computed for the extragalactic O stars investigated by Tramper et al. (2011, ApJ, 741, 8 - TSKK). For one early-type $\mathrm{O}$ star, computed and observed rates agree within errors. However, for two late-type $\mathrm{O}$ stars, theoretical mass-loss rates underpredict observed rates by $\sim 1.6$ dex, far exceeding observational errors. A likely cause of the discrepancy is overestimated observed rates due to the neglect of wind-clumping. A less likely but intriguing possibility is that, in observing $\mathrm{O}$ stars with $Z / Z_{\odot} \sim$ $1 / 7$, TSKK have serendipitously discovered an additional mass-loss mechanism not evident in the spectra of Galactic O stars because of their powerful radiation-driven winds. Constraints on this unknown mechanism are discussed. In establishing that the discrepancies, if real, are inescapable for purely radiation-driven winds, failed searches for high- $J$ solutions are reported and the importance stressed of a numerical technique that cannot spuriously create or destroy momentum.

The $Z$-dependences of the computed rates for $Z / Z_{\odot} \in(1 / 30,2)$ show significant departures from a single power law, and these are attributed to curve-of-growth effects in the differentially expanding reversing layers. The best-fitting power-law exponents range from $0.68-0.97$.
\end{abstract}

Key words. stars: early-type - stars: mass-loss - stars: winds, outflows

\section{Introduction}

By observing $\mathrm{O}$ stars in nearby dwarf galaxies with metallicities $(Z)$ below that of the Small Magellanic Cloud, Tramper et al. (2011, hereafter TSKK) have extended the range of $Z$ over which $\mathrm{O}$-star winds have been detected and analysed. The resulting mass-loss rates $(\Phi)$ for $Z / Z_{\odot} \sim 1 / 7$ are surprisingly high, leading the authors to question the Vink et al. (2001) scaling law $\Phi \propto Z^{\alpha}$ with $\alpha=0.69 \pm 0.10$.

Because of this result's importance for the later evolutionary phases of massive stars and perhaps also for the theory of stellar winds, models computed with the TSKK stars' effective temperatures $\left(T_{\text {eff }}\right)$ and surface gravities $(g)$ are of interest in order to quantify discrepancies without relying on scaling laws. In addition, the validity of power-law scaling merits investigation. Accordingly, in this paper, mass fluxes $J=\Phi / 4 \pi R^{2}$ are computed for a wide range of $Z$ at several points in $\left(T_{\mathrm{eff}}, g\right)$-space.

Throughout this paper, the units of $J$ and $\Phi$ are $\mathrm{gm} \mathrm{s}^{-1} \mathrm{~cm}^{-2}$ and $\odot \mathrm{yr}^{-1}$, respectively. In these units,

$\log \Phi=\log J+2 \log R / R_{\odot}-3.015$.

\section{Mass fluxes}

In this section, the assumptions and procedures adopted when computing $J$ from the theory of moving reversing layers (RL's) are briefly reviewed, as are the curve-of-growth (CoG) effects that control $J$ 's dependence on $Z$.

\subsection{Assumptions}

The fundamental assumption is that the $\Phi$ of a stationary, radiation-driven wind is determined by the constraint of regularity at the sonic point - i.e., that the transonic flow makes a smooth (analytic) transition from sub- to supersonic velocities. This assumption dates back to Lucy \& Solomon's (1970, LS70) primitive theory of dynamic RL's and retains its role in updated versions (Lucy 2007, 2010a,b, hereafter L07, L10a and L10b, respectively).

Additional assumptions are: 1) plane-parallel, isothermal flow; 2) continuum emission occurs only at the lower boundary of the Schuster-Schwarzschild RL and has the frequency distribution of the TLUSTY model atmosphere with the prescribed $T_{\text {eff }}, g$ and $Z$ (Lanz \& Hubeny 2003); 3) line formation occurs by non-coherent scattering with complete redistribution in the fluid frame; 4) line broadening is due radiation damping and Doppler broadening with microturbulent velocity $v_{t}=10 \mathrm{~km} \mathrm{~s}^{-1}$;5) the RL's upper boundary condition of no incoming radiation is imposed at velocity $v=5 a$, where $a$ is the isothermal sound speed.

\subsection{Procedures}

This multi-line transfer problem in a differentially expanding RL is treated with a Monte Carlo (MC) technique. This has the considerable merit of coding simplicity but has the drawback that $\tilde{g}_{\ell}$, the MC estimate for the contribution of lines to the radiative acceleration, is not analytic. This poses the problem of how to determine the eigenvalue $J$ from the regularity condition

$g_{\text {eff }}=g_{*}-g_{\ell}=0$ at $v=a$

where $g_{*}=g-g_{\mathrm{e}}$ and $g_{\mathrm{e}}$ is the radiative acceleration due to electron scattering.

The procedure developed in L07 and L10b replaces this point constraint by an integral constraint $Q_{1,2}=0$, which is here conveniently rewritten as

$\Delta_{1,2}=\int_{x_{1}}^{x_{2}} g_{\ell} \mathrm{d} x=\left|\frac{1}{2} v^{2}-a^{2} \ln v+g_{*} x\right|_{x_{1}}^{x_{2}}$ 
where the heights $x_{1}$ and $x_{2}$ correspond to $v / a \approx 1 / 2$ and $\approx 2$, respectively. The interval $\left(x_{1}, x_{2}\right)$ includes the sonic point but excludes zones where the solution is affected by inexact boundary conditions.

A MC simulation with $\mathcal{N}$ indivisible photon packets is carried out in a model RL derived from the trial solution: $g_{\ell}=g_{i}^{\ell}$ at $v_{i}$ for $i=1,2, \ldots, k, \ldots, I$ with $g_{k}^{\ell}=g_{*}$ at $v_{k}=a$. The resulting estimates $\tilde{g}_{\ell}$ then allow the left-hand side of Eq. (3) to be evaluated numerically, thereby testing if the equation is satisfied. With trial-and-error adjustments of $g_{i}^{\ell}$ for $i \neq k$, sequences of such simulations allow the eigenvalue $J$ to be determined (L10a,b).

This numerical procedure achieves consistency with the posed integro-differential problem in the limits $I \rightarrow \infty$ and $\mathcal{N} \rightarrow \infty$, provided that the iterative adjustments of $J$ and the $g_{i}^{\ell}$ have resulted in exact agreement between $\tilde{g}_{\ell}$ and $g_{i}^{\ell}$ throughout the RL.

The main weakness at present of this technique is its reliance on trial-and-error adjustment. In consequence, each solution demands a large commitment of the investigator's time. A reliable iterative algorithm is needed if large numbers of solutions are required.

Because the MC quanta are indivisible, none are created or destroyed within the RL. Accordingly, this MC technique does not spuriously create or destroy momentum. The sonic-point momentum flux $J a$ therefore derives from identifiable physical processes.

\subsection{Curve-of-growth effects}

Classically, the CoG refers to static RL's and relates the emergent line profile and its equivalent width to $N$, the absorbing ion's column density above the photosphere. However, the physical effects underlying the $\mathrm{CoG}$ are also relevant (LS70) to the $J$ that can be radiatively-driven through the sonic point since $g_{\ell}$ at $v=a$ depends on the co-moving radiation field, and this reflects line formation throughout the RL, including the quasi-static layers close to the photosphere.

For simplicity, consider an outflow driven by one line from an atmosphere with continuum absorption due only to $\mathrm{H}$ and imagine varying $Z$ with fixed $T_{\text {eff }}, g$. We follow the discussion in LS70 for the $\lambda 1548 \AA$ component of the C IV doublet when the dominant ion is $\mathrm{C}$ III. For vanishingly small $Z$ - i.e., negligible line formation, $g_{\ell}^{\dagger} \propto n_{1}^{\dagger}$, the ground state number density at $v^{\dagger}=a$. On the assumption of an ionization balance between radiative recombinations and photoionizations, $n_{1}^{\dagger} \propto n_{C}^{\dagger} / n_{\mathrm{e}}^{\dagger} \propto$ $Z / \rho^{\dagger}$. Accordingly, the sonic point constraint $g_{\ell}^{\dagger}=g_{*}$ is obeyed at density $\rho^{\dagger} \propto Z$, whence $J=\rho^{\dagger} v^{\dagger} \propto Z$.

In this weak-line limit, $J \propto Z$ because of increasing $N$. This effect is seen in Fig. 2 of LS70 at $\log T_{\text {eff }} \approx 4.55$ and at $\approx 4.25$, where increasing $N$ is due to shifting ionization balance as $T_{\text {eff }}$ increases.

When increasing $Z$ invalidates the weak-line limit, line formation reduces the co-moving flux seen by ions at $v \sim a$ and so $J(Z)$ flattens, reaching a maximum at $J \sim J_{*}=\sigma T_{\text {eff }}^{4} / c^{2}$, when all the photon momentum accessible with one line is converted into momentum of the transonic flow (LS70). In terms of the dimensionless rate $\phi=\Phi c^{2} / L$, this maximum corresponds to $\phi \sim 1$.

When increasing $Z$ brings about the strong-line limit, corresponding to a line with a saturated Doppler core and welldeveloped damping wings, the photon momentum available at $v \sim a$ is drastcally reduced, as therefore is $J$. This effect is seen in Fig. 2 of LS70 at $\log T_{\text {eff }} \approx 4.5$ In this strong-line limit, the accessible photon momentum in the continuum is transferred to quasi-stationary gas $\left(v^{2} \ll a^{2}\right)$ deep in the RL where it merely increases $\mathrm{H}_{\rho}$, the local scale height.

With more than one line, the above CoG effects are superposed. However, in regions of high line-density, effects of overlapping line formation come into play. These defy simple interpretation and are the main reason for adopting MC methods.

\section{Models with varying $Z$}

In this section, models are computed at selected points in $\left(T_{\text {eff }}\right.$, $g, Z)$-space.

\subsection{Parameters}

Because the RL's require input from the OSTAR2002 models of Lanz \& Hubeny (2003), their grid somewhat restricts the parameter choices made here. We adopt the TLUSTY labelling scheme, so that, for example, model Gt400g300 has $T_{\text {eff }}=40.0 \mathrm{kK}, \log g=3$ and Galactic metallicity. In order to test scaling laws over the full range of metallicites found in the local Universe, a sequence of models with letter codes C, G, L, S, T and $\mathrm{V}$ are computed at each selected point $\left(T_{\mathrm{eff}}, g\right)$. These correspond, respectively, to $Z / Z_{\odot}=2,1,1 / 2,1 / 5,1 / 10$ and $1 / 30$, a range of 60 in metallicity.

With regard to $T_{\text {eff }}$ and $g$, points in the OSTAR2002 grid are chosen to match the best-fit parameters in Table 2 of TSKK. The chosen points and the stars matched within errors are:

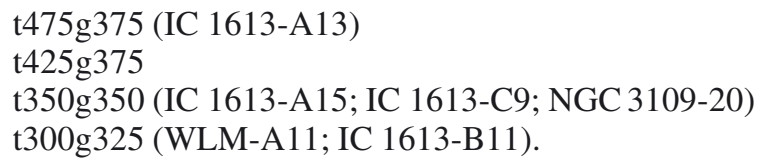

The $425 \mathrm{~g} 375$ sequence is included to avoid a large gap in $T_{\text {eff }}$.

All models have $v_{t}=10 \mathrm{~km} \mathrm{~s}^{-1}$. This canonical value is consistent with the highly uncertain TSKK estimates. Note that well-determined values of $v_{t}$ for Galactic $\mathrm{O}$ stars (e.g., Bouret et al. 2005) derive from UV spectra, which are not available for the TSKK stars.

\subsection{Model sequencies}

Values of $J$ for the four sequencies are given in Table 1. The additional tabulated quantities are:

Col. 4: $\phi$, the mass-loss rate in units of $L / c^{2}$.

Since $\phi$ has a maximum value $\sim 1$ with one driving line (LS70), $\phi$ is the equivalent number of effective non-overlapping lines.

Col. 5: $\eta$, the percentage of the photon packets escaping across the RL's upper boundary that have not undergone a line scattering event within the RL.

Since the MC quanta emitted at the lower boundary sample the continuum flux distribution of the TLUSTY model, a high value of $\eta$ indicates that much of this emission occurs in relatively line-free frequency intervals. Conversely, a low value indicates effective line-blocking of the star's continuum.

Col. 6: $\zeta$, the percentage of $\Delta_{1,2}-$ see Eq. (3) - contributed by lines of $\mathrm{Fe}$ and $\mathrm{Ni}$.

This quantity measures the importance of $\mathrm{Fe}$ and $\mathrm{Ni}$ relative to $\mathrm{C}$, $\mathrm{N}, \mathrm{O}, \mathrm{Ne}, \mathrm{Si}$ and $\mathrm{S}$ in accelerating matter through the sonic point. Note that the MC estimator for $g_{\ell}-$ Eq. (10) in L07 - readily allows the contributions of individual lines to be extracted. 
L. B. Lucy: O-star mass-loss rates

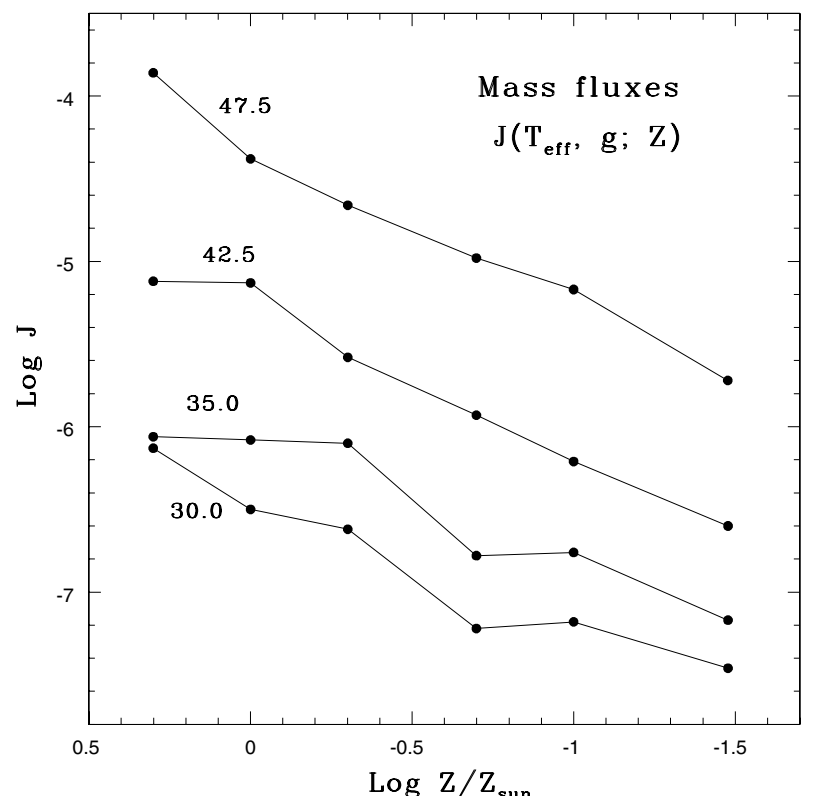

Fig. 1. Mass fluxes $J$ from Table 1 plotted against metallicity $Z / Z_{\odot}$. The curves are labelled with the sequencies' $T_{\text {eff }}(\mathrm{kK})$.

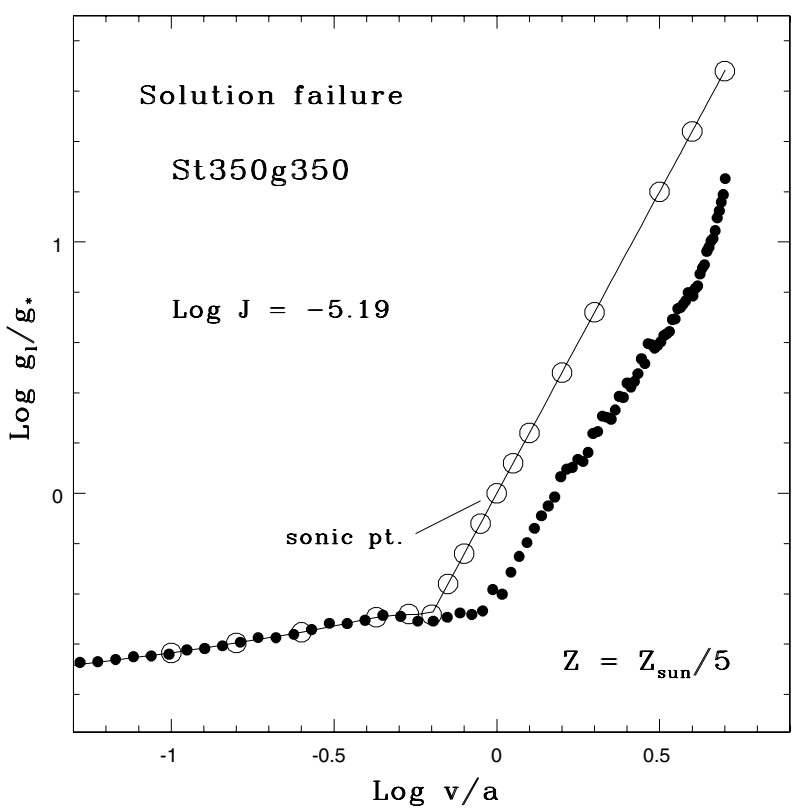

Fig. 2. Failed solution for $S t 350 g 350$ when $J=-5.19$ dex. The open circles are the $g_{i}^{\ell}$ for $i=1,2, \ldots, k, \ldots, I$ from which the model RL is derived. The filled circles are the MC estimates $\tilde{g}_{\ell}$. The sonic point $(i=k)$ is indicated.

\subsection{Dependence of $\mathrm{J}$ on metallicity}

In Fig. 1 , the $J$ 's given in Table 1 are plotted against $Z / Z_{\odot}$. These $\log -\log$ plots show significant departures from linearity and even include departures from the expected monotonic decline with decreasing $Z$.

The quantities $\eta$ and $\zeta$ provide a qualitative understanding of the $J$ 's dependence on $Z$. In the main, we see that $J$ increases with $Z$ but that $\eta$ decreases. Thus, the enhanced $J$ and $\phi$ can be attributed to the shrinking of line-free frequency intervals as weak lines become significant absorbers.

The importance of CoG effects is illustrated by $\zeta$. The spectra of $\mathrm{Fe}$ and $\mathrm{Ni}$ ions have numerous weak permitted lines arising
Table 1. Computed mass fluxes $J\left(\mathrm{gm} \mathrm{s}^{-1} \mathrm{~cm}^{-2}\right)$.

\begin{tabular}{lccccc}
\hline \hline Seq. & $Z / Z_{\odot}$ & $\log J$ & $\phi$ & $\eta(\%)$ & $\zeta(\%)$ \\
\hline \multirow{4}{*}{$t 475 g 375$} & 2 & -3.86 & 429.8 & 37.8 & 80.0 \\
& 1 & -4.38 & 129.8 & 47.2 & 81.5 \\
& $1 / 2$ & -4.66 & 68.1 & 52.4 & 80.3 \\
& $1 / 5$ & -4.98 & 32.6 & 58.6 & 69.9 \\
& $1 / 10$ & -5.17 & 21.1 & 58.0 & 58.7 \\
& $1 / 30$ & -5.72 & 5.9 & 73.9 & 23.6 \\
\hline \multirow{5}{*}{$t 25 g 375$} & 2 & -5.12 & 36.9 & 62.8 & 89.4 \\
& 1 & -5.13 & 36.0 & 65.4 & 86.4 \\
& $1 / 2$ & -5.58 & 12.8 & 69.8 & 64.6 \\
& $1 / 5$ & -5.93 & 5.7 & 74.5 & 38.7 \\
& $1 / 10$ & -6.21 & 3.0 & 78.5 & 21.4 \\
& $1 / 30$ & -6.60 & 1.2 & 84.6 & 9.9 \\
\hline & 2 & -6.06 & 9.2 & 61.0 & 64.6 \\
& 1 & -6.08 & 8.8 & 66.1 & 53.3 \\
& $1 / 2$ & -6.10 & 8.4 & 70.0 & 50.6 \\
& $1 / 5$ & -6.78 & 1.8 & 76.4 & 23.2 \\
& $1 / 10$ & -6.76 & 1.8 & 79.6 & 14.6 \\
& $1 / 30$ & -7.17 & 0.7 & 81.8 & 5.7 \\
\hline \multirow{5}{*}{ 300g325 } & 2 & -6.13 & 14.5 & 59.3 & 50.4 \\
& 1 & -6.50 & 6.2 & 64.6 & 33.2 \\
& $1 / 2$ & -6.62 & 4.7 & 69.7 & 21.8 \\
& $1 / 5$ & -7.22 & 1.2 & 76.2 & 5.2 \\
& $1 / 10$ & -7.18 & 1.3 & 79.9 & 4.1 \\
$1 / 30$ & -7.46 & 0.7 & 85.9 & 2.1 \\
\hline & & & & &
\end{tabular}

Table 2. Power law exponents $\alpha$.

\begin{tabular}{lcc}
\hline \hline Seq. & $\alpha \pm \sigma_{\alpha}$ & $\sigma_{\log J}$ \\
\hline$t 475 g 375$ & $0.97 \pm 0.07$ & 0.10 \\
$t 425 g 375$ & $0.89 \pm 0.07$ & 0.10 \\
$t 350 g 350$ & $0.68 \pm 0.11$ & 0.17 \\
$t 300 g 325$ & $0.75 \pm 0.10$ & 0.15 \\
\hline
\end{tabular}

from metastable levels. In contrast, the line-driving contributed by light and intermediate mass elements is predominantly from strong ground-state resonance transitions. As $Z$ increases, these ground-state transitions saturate, and their momentum tranfers then occur deep in the RL, increasing $\mathrm{H}_{\rho}$ but contributing little to $J$. Because of this effect, the relative contribution of $\mathrm{Fe}$ and $\mathrm{Ni}$ to $\Delta_{1,2}$ increases dramatically with $Z$.

\subsection{Power-law exponents $\alpha$}

Conventionally, the $Z$-dependence of $\Phi$ is approximated as a power law, so that

$\log J=\log J_{G}+\alpha \log Z / Z_{\odot}$.

Although Fig. 1 shows significant departures from this behaviour, least squares fits of Eq. (4) to the data have been carried out and are given in Table 2 . The intercepts $\log J_{G}$ are not reported since they do not supersede the individual $J$-values for the $G$-models.

In view of the significant departures from power-law behaviour, the 3-D function $J\left(T_{\text {eff }}, g ; Z\right)$ will eventually have to be tabulated so that the evolution of massive stars can be followed as a function of initial metallicity.

\section{Comparisons with observed $\Phi$ 's}

In this section, the $\Phi$ 's derived by TSKK for three of their stars are compared to the predictions of Sect. 3 . The stars chosen are 
the one with high $T_{\text {eff }}$ and the two late-type $\mathrm{O}$ stars with the most accurate $\Phi$ 's.

\subsection{IC 1613-A13}

For this star, TSKK estimate $T_{\text {eff }}(\mathrm{kK})=47.6_{-4.95}^{+4.73}$ and $\log g=3.73_{-0.22}^{+0.13}$ and so the appropriate sequence from Table 1 is $t 475 \mathrm{~g} 375$. The metallicity of IC 1613 is $Z / Z_{\odot} \approx 1 / 7$, which is bracketed by the models with $Z / Z_{\odot}=1 / 5$ and $1 / 10$. Logarithmic interpolation gives $J=-5.07$ dex. Accordingly, with $R / R_{\odot}=$ 11.4 from TSKK, $\Phi=4 \pi R^{2} J=-5.97$ dex. This is to be compared with the TSKK estimate $\Phi=-6.26_{-0.50}^{+0.45}$ dex. Thus theory overpredicts observation by 0.29 dex, but this is well within observational uncertainty and so there is no conflict.

\subsection{NGC 3109-20}

For this star, TSKK derive $T_{\text {eff }}(\mathrm{kK})=34.2_{-3.05}^{+4.70}$ and $\log g=$ $3.48_{-0.40}^{+0.37}$ and so the appropriate sequence is $t 350 \mathrm{~g} 350$. The metallicity of $\mathrm{NGC} 3109$ is $Z / Z_{\odot} \approx 1 / 7$. Logarithmic interpolation gives $J=-6.77$ dex. Accordingly, with $R / R_{\odot}=24.7$ from TSKK, $\Phi=-7.00 \mathrm{dex}$. This is to be compared to the TSKK estimate that $\Phi=-5.41_{-0.35}^{+0.25}$ dex. Thus theory underpredicts observation by $1.59 \mathrm{dex}$, far beyond the observational uncertainty.

In dimensionless terms, the TSKK rate is $\phi=76$, indicating the number of effective driving lines required to accelerates the corresponding $J$ through the sonic point. Inspection of Table 1 shows that $\phi=76$ is only achieved for $T_{\mathrm{eff}} \gtrsim$ 45.0 and $Z / Z_{\odot} \gtrsim 1 / 2$, a domain excluded by TSKK's spectroscopic analysis. Moreover, the interpolated $\eta$ for this model is $\approx 78$ per cent, showing that there are extensive frequency intervals not contributing to line driving of the transonic flow.

\subsection{WLM-A11}

For this star, TSKK derive $T_{\text {eff }}(\mathrm{kK})=29.7_{-2.75}^{+2.45}$ and $\log g=$ $3.25_{-0.19}^{+0.29}$ and so the appropriate sequence is $t 300 \mathrm{~g} 325$. The metallicity of WLM is again $Z / Z_{\odot} \approx 1 / 7$. In this case, interpolation gives $J=-7.20$ dex. Accordingly, with $R / R_{\odot}=29.8$ from TSKK, $\Phi=-7.27$ dex. This is to be compared with the TSKK estimate that $\Phi=-5.56_{-0.30}^{+0.20}$ dex. Thus theory underpredicts observation by $1.71 \mathrm{dex}$, far outside the observational uncertainty.

In dimensionless terms, the TSKK rate is $\phi=66$ Thus, as for NGC 3109-20, this is only achieved for $T_{\text {eff }} \gtrsim 45.0$ and $Z / Z_{\odot} \gtrsim$ $1 / 2$, a domain excluded by TSKK's spectroscopic analysis. The interpolated $\eta$ for this model is also $\approx 78$ per cent, thus showing again that there are extensive frequency intervals that cannot be tapped to sustain a high $J$.

\section{Discussion}

In this section, the severe conflict between theory and observation for the two late-type $\mathrm{O}$ stars is discussed. For clarity, the two extremes are considered.

\subsection{Observed rates in error}

The possible causes of overestimation of observed rates are discussed in detail by TSKK. As they fully recognize, a likely cause is wind clumping. Their estimates of $\Phi$ derive from the partial filling-in of the He II $\lambda 4686 \AA$ and $\mathrm{H} \alpha$ absorption lines by wind emission - see their Fig. 2. Now, it has long been suspected that the instability of line-driven winds will lead to a clumpy outflow and hence enhanced emission in exactly these lines (Lucy 1975). Moreover, the standard diagnostic code CMFGEN (Hillier \& Miller 1999) includes an adjustable clumping factor that demonstrably improves fits to observed spectra (e.g., Bouret et al. 2008). Nevertheless, the model wind (Puls et al. 2005) that SDKK use to fit the data does not allow for clumpiness. Accordingly, one suspects that, if clumpiness were included, their genetic algorithm (Mokiem et al. 2005) would find a wide range of acceptable solutions: from high $\Phi$ 's with weak clumping to low $\Phi$ 's with strong clumping - i.e., degeneracy with respect to these two parameters.

In view of the limited information content concerning winds in the optical spectra of O stars, the $\Phi$ 's of the TSKK stars are likely to remain highly uncertain until UV spectra can be obtained. However, in the meantime, as a control experiment, the TSKK methodology should be applied to optical spectra of Galactic O stars that already have analysed UV spectra. If the $\Phi$ 's of Galactic $O$ stars with similar spectral types to NGC 3109-20 and WLM-A11 can thus be derived with reasonable accuracy purely from their optical spectra, then the high TSKK $\Phi$ 's for these low- $Z$ extragalactic O stars would be more convincing.

\subsection{Theoretical rates in error}

The accuracy of the eigenvalues $J$ is discussed in Sect. 4.2 of L10b, from which it follows that errors $\sim 1.6$ dex would appear to be excluded. However, there is no proof that the eigenvalue $J$ is unique. Accordingly, a search for a second, high- $J$ solution has been carried out for $\operatorname{St} 350 \mathrm{~g} 350\left(Z=Z_{\odot} / 5\right)$. Thus, as described in Sect. 2.2, the vector $g_{i}^{\ell}$ for $i \neq k$ is iteratively corrected with the aim of achieving dynamical consistency by matching the MC estimates $\tilde{g}_{\ell}$. But now $J$ is fixed at -5.19 dex, a value obtained by adding the discrepancy of 1.59 dex found in Sect. 4.2 for NGC 3109-20 to the eigenvalue $J=-6.78 \mathrm{dex}$ from Table 1 (cf. Sect. 3.3 in L10a).

Figure 2 illustrates the limited degree of consistency achieved when the search was abandoned. This may be compared with Fig. 2 in L10b, where the successful solution for $G t 400 \mathrm{~g} 375$ is plotted. In that case, $J$ was not fixed but instead was iteratively corrected to obtain $\Delta_{1,2}=0$.

Figure 2 shows that dynamical consistency is achievable for $v / a \lesssim 0.6$ but fails at higher velocities. Evidently, the constraint that the dynamical RL satisfies the regularity constraint (Eq. (2)) precludes adjustment into agreement with $\tilde{g}_{\ell}$ as the sonic point is approached. Accordingly, the inescapable conclusion is that a second solution with $\Delta \log J \sim 1.6$ does not exist.

An identical experiment for $S t 300 \mathrm{~g} 325$ with $J$ fixed at $-7.22+1.71=-5.11 \mathrm{dex}$ also ended in a failure similar to Fig. 2.

The MC code cannot match these imposed sonic point momentum fluxes $\mathrm{Ja}$ because there are no physical processes available to provide these fluxes and the use of indivisible MC quanta precludes the spurious creation of momentum (Sect. 2.2).

\subsection{An additional mass-loss mechanism?}

If the $\Phi$ estimates of TSKK are accurate, then the above elimination of high- $J$ solutions implies that an additional acceleration mechanism is operating besides radiative driving. This is perhaps an unwelcome possibility, but where better to discover an additional mechanism than in the spectra $\mathrm{O}$ stars with low-Z's? 
For steady flow, Fig. 2 shows that this unknown mechanism must provide an outward force per gm of $\sim 0.4 \mathrm{~g}$ at $v=a$ in order to make up for $\tilde{g}_{\ell}$ 's shortfall in satsfying Eq. (2). But extra driving is not needed at higher velocities. For $v / a \gtrsim 1.5$, Fig. 2 shows that a net outward acceleration is provided by $\tilde{g}_{\ell}$. This is a consequence of matter being Doppler-shifted out of the absorption lines formed in the RL's quasi-static layers.

The possibility that an extra mechanism may be required only in the neighbourhood of the sonic point prompts the thought that small-scale, stochastic expulsions may be accelerating blobs of photospheric matter to $v / a \gtrsim 1.5$, whereupon line-driving takes over and a high- $\Phi$ supersonic wind ensues.

A strong constraint on this unkown mechanism is provided by the existence of a weak-wind domain for Galactic $\mathrm{O}$ stars (Marcolino et al. 2009, M09). From Table 3 in M09, we see that, observationally, this domain's extent is $T_{\text {eff }}(\mathrm{kK}) \in(31,34)$ and $\log g \in(3.6,4.0)$. Thus, in $\left(T_{\text {eff }}, g\right)$-space, the two latetype $\mathrm{O}$ stars of TSKK are contiguous to the weak-wind domain, whose reality is solidly-based on UV spectra and is partially understood theoretically (L10a,b). Evidently, the unknown mechanism does not operate in the weak-wind domain. Accordingly, it must be narrowly focused in parameter space, perhaps also excluding $Z / Z_{\odot} \sim 1$.

\section{Conclusion}

The aim of this paper has been to test the theory of moving RL's at metallicities other than Galactic. The stimulus was the high empirical $\Phi$ 's reported recently by TSKK for extragalactic
O stars with $Z / Z_{\odot} \approx 1 / 7$ and the doubts they raised about the Vink et al. (2001) scaling law.

Although agreement within errors is found at $T_{\text {eff }}(\mathrm{kK}) \sim$ 47.5, discrepacies $\sim 1.6-1.7 \mathrm{dex}$ are found for two late type O stars with $T_{\text {eff }}(\mathrm{kK}) \sim 30-35$. If these discrepancies are not due to wind clumping, an effect not included by TSKK, then an additional mass-loss mechanism is necessary, perhaps operating only at low velocities and in a restricted part of $\left(T_{\mathrm{eff}}, g, Z\right)$-space (Sect. 5.3). What this mechanism might be is an open question.

Most probably, wind-clumping is the solution to these discrepancies, but the proof may not come until we can obtain spectra in the UV.

\section{References}

Bouret, J.-C., Lanz, T., \& Hillier, D. J. 2005, A\&A, 438, 301

Bouret, J.-C., Lanz, T., Hillier, D. J., \& Foellmi, C. 2008, in Clumping in Hot Star Winds, ed. W.-R. Hamann, A. Feldmeier, \& L. M. Oskinova (Potsdam: Univ. - Verl.)

Hillier, D. J., \& Miller, D. L. 1999, ApJ, 519, 354

Lanz, T., \& Hubeny, I. 2003, ApJS, 146, 417

Lucy, L. B. 1975, Mem. Soc. R. Sci. Liège, 8, 359

Lucy, L. B. 2007, A\&A, 468, 649 (L07)

Lucy, L. B. 2010a, A\&A, 512, A33 (L10a)

Lucy, L. B. 2010b, A\&A, 524, A41 (L10b)

Lucy, L. B., \& Solomon, P. M. 1970, ApJ, 159, 879 (LS70)

Marcolino, W. L. F., Bouret, J.-C., Martins, F., et al. 2009, A\&A, 498, 837 (M09)

Mokiem, M. R., de Koter, A., Puls, J., et al. 2005 A\&A, 441, 711

Puls, J., Urbaneja, M. A., Venero, R., et al. 2005, A\&A, 435, 669

Tramper, F., Sana, H., de Koter, A., \& Kaper, L. 2011, ApJ, 741, 8 (TSKK)

Vink, J. S., de Koter, A., \& Lamers, H. J. G. L. M. 2001, A\&A, 369, 574 